\title{
Mobility control of ceramic grain boundaries and interfaces
}

\author{
I-Wei Chen \\ Department of Materials Science and Engineering, University of Michigan, Ann Arbor, MI 48109 (USA)
}

\begin{abstract}
Grain boundary mobility and grain-liquid boundary mobility in ceramics vary vastly from material to material. Their characteristics are sensitive to the crystal structure, the nature of bonding, orientation, stoichiometry and composition. More directly, mobility can be lowered by decreasing the interfacial energy and anisotropy or increasing solute drag. liquid viscosity, particle pinning and grain interlocking. Judicious doping and scavenging emerge as especially effective methods for mobility control. Static and dynamic grain growth data of zirconia, alumina, and silicon nitride are cited to support the above proposition.
\end{abstract}

\section{Introduction}

The motion of grain boundary and grain-liquid interface is an important step in microstructural development. In solid state sintering, a low grain boundary mobility allows pores to remain attached to the grain boundary and to continue rapid shrinkage via grain boundary diffusion. This realization has led ceramists to search for additives that aid sintering through controlled grain growth [1]. In recent years, the interest in grain growth has continued unabated amid the activities of nanocrystalline ceramics [2], superplastic ceramics [3], grain-aligned high $T_{\mathrm{c}}$ superconductors [4], in situ toughened silicon nitride [5], and transformation toughened zirconia [6]. In all of the above endeavors, the ceramists' ability to enhance or suppress grain boundary and grain-liquid interface motion is critical to the successful rendering of the desired microstructures and properties. For high temperature plasticity, which is the focus of the present workshop, the tendency for the stressed interface to sustain localized deformation and for highly mobile interfaces to undergo enhanced motion and effect dynamic microstructural evolution is obviously a matter of special interest.

The characteristics and the methods for controlling interface motion in ceramics vary considerably from material to material. Nevertheless, from theoretical considerations and the large database of technologically important ceramic systems, we have tentatively identified the most important factors pertinent to mobility control. These include interfacial energy, interface anisotropy, solute drag, liquid viscosity, particle pinning, and grain interlocking. The intent of the present contribution is to review the recent research on zirconia, alumina, and silicon nitride to provide relevant examples of these controlling factors. In this context we paid special attention to cases involving high temperature deformation.

There are large differences in the grain boundary and interface behavior of ceramics, even among the above three ceramics. Some of these differences can be attributed to the basic nature of bonding and structure of the crystal. In particular, the extent of stoichiometry, solubility and anisotropy appears to be important. These considerations also extend to the interface itself. For example, whether the interface is charged or not figures prominently in mobility. The other relevant consideration is the composition of the grain boundary. In particular, the common presence of siliceous impurity and the immiscibility between glassy silica and many ceramics leaves the distinct possibility of a grain boundary glassy phase remaining after sintering. Indeed, silicate ceramics and nitrides are often processed with a liquid phase. Moreover, in the latter practice, a series of non-equilibrium reactions usually takes place during which a mechanical force is often superimposed (e.g. in hot-pressing). Lastly, since ceramics are hard and have limited slip systems and few dislocations, the elastic energy associated with the interface or growth may not be easily relieved. Some mechanical effects on interface motion are thus expected. For each ceramic, these basic features and processing complexities have to be duly recognized. 


\section{Zirconia}

Zirconia has a crystal structure closely related to that of fluorite. It usually appears in either tetragonal or cubic forms, and their respective phase stability depends on the amount and type of cation additives. These additives are typically of a lower valence, such as $\mathrm{Mg}$ and $\mathrm{Y}$, and their amount varies from $1 \%$ to $2 \%$ in tetragonal zirconia to $8 \%$ to $20 \%$ in cubic zirconia. It also forms a complete solid solution with other fluorite structural oxides such as $\mathrm{CeO}_{2}$. Oxygen vacancies are the dominant defect species in all zirconia and provide a fast diffusion mechanism for oxygen. Its grain shape is usually equiaxed even in the tetragonal phase, which has only a small tetragonality.

Grain boundary mobility has been measured in several tetragonal zirconia and cubic zirconia $[7,8]$, see Fig. 1. They vary by two orders of magnitude at $1500{ }^{\circ} \mathrm{C}$ and more at lower temperatures. In the case of tetragonal zirconia, the controlling factor of grain boundary migration has been identified as solute drag [8]. This is supported by (a) direct confirmation of solute segregation at the grain boundary by Auger and electron spectroscopy for chemical analysis (ESCA), (b) negative concentration dependence of the mobility, and (c) a relatively high activation energy comparable with that for lattice diffusion of solute. In contrast, the activation energy of cubic zirconia is much lower and might be identified as that for grain boundary diffusion [7]. In general, the lower the grain boundary mobility, the higher the activation energy.

The cause for grain boundary segregation in tetragonal zirconia has been attributed to the charge effect [8]. This may be understood by considering chargedistribution near the grain boundary, as shown in Fig. 2. Usually we assume the cation and anion vacancy

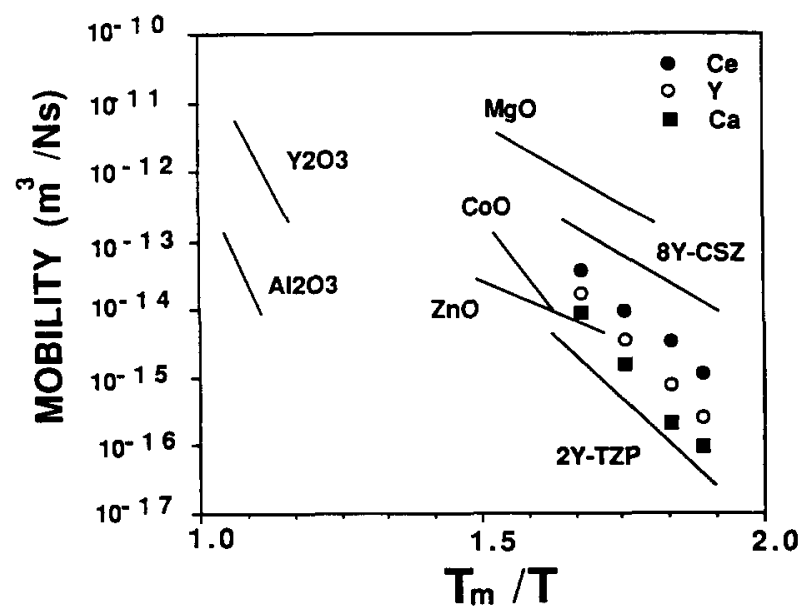

Fig. 1. Grain boundary mobility in several tetragonal and cubic zirconia $[7,8]$. Reference data for other ceramics are also indicated. concentrations, $\left[\mathrm{V}_{\mathrm{o}}\right]$ and $\left[\mathrm{V}_{\mathrm{Z}_{\mathrm{r}}}^{\prime \prime \prime}\right]$, are equilibrium, determined by the reaction of the Schottky defect which has a formation energy of the order of $5 \sim 7 \mathrm{eV}$. These concentrations are typically very low. When aliovalent dopants such as $\mathrm{Ca}^{2+}$ are introduced, charge consideration dictates that the oxygen vacancy concentration in the bulk is approximately equal to the dopant concentration which is much higher than $\left[\mathrm{V}_{\mathrm{o}}^{*}\right]$ at the grain boundary. ( $\left[\mathrm{V}_{\mathrm{Zr}}^{\prime \prime \prime \prime}\right]$ is very small since it is of the same effective charge as $\mathrm{Ca}^{2+}$.) The gradient of $\left[\mathrm{V}_{\mathrm{o}}^{*}\right]$ sets up a space charge potential which attracts $\mathrm{Ca}^{2+}$ to the grain boundary. The total amount of solute segregation $\Delta C$ can be shown to be proportional to $Z^{3 / 2} C_{0}^{1 / 2}[9]$ where $Z$ is the difference in the valence of the dopant and the host cation, and $C_{0}$ is the bulk concentration of solute. Thus, the higher the solute concentration and the larger the valence difference, the more segregation is expected. When a grain boundary migrates, it must drag these segregated solutes along. This increases the resistance to grain boundary motion and lowers its mobility.

Solute drag as a result of segregation is proportional to the same charge and concentration factors. In addition, since the solute movement is achieved by lattice diffusion, the drag increases with $D_{\text {solute }}{ }^{-1}$, where $D_{\text {solute }}$ is the lattice diffusivity of the solute. Inasmuch as large solutes often have lower diffusivity in tetragonal zirconia, larger solutes are more effective grain growth inhibitors. Indeed, among all the solutes of tetragonal zirconia, $\mathrm{Ca}$, which has the lowest valence and one of

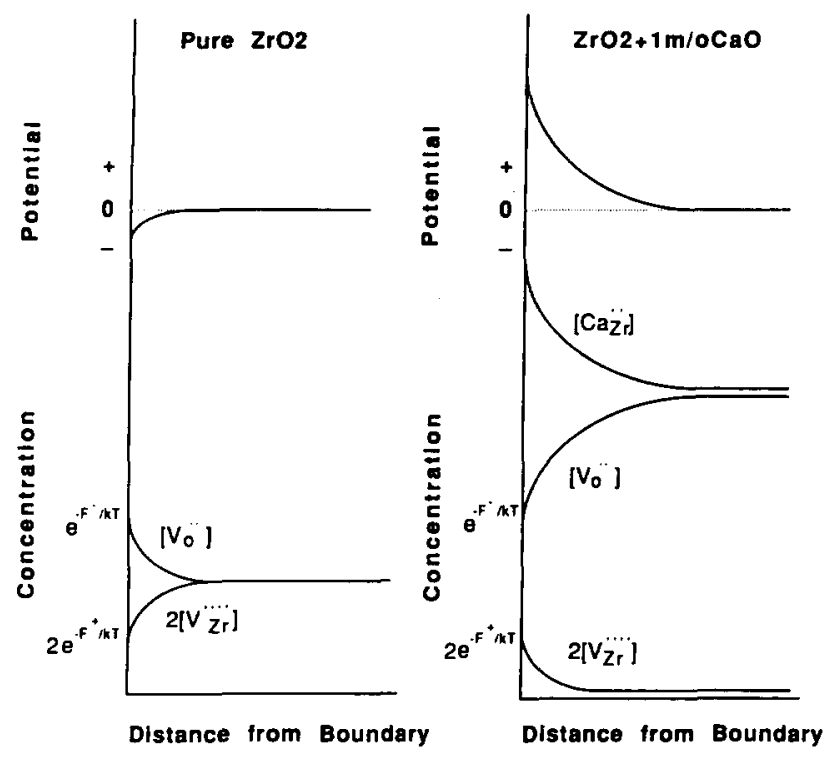

Fig. 2. Schematic variations of electrostatic potential and defect concentrations with distance from grain boundary. Opposite grain boundary charges are developed in the intrinsic (pure $\left.\mathrm{ZrO}_{2}\right)$ and in the extrinsic $\left(\mathrm{ZrO}_{2}+1 \mathrm{~mol} \% \mathrm{CaO}\right)$ regimes. $F^{+}$is the formation energy of $\mathrm{V}_{\mathrm{Z}_{r}}^{\prime \prime \prime \prime}, F^{-}$is the formation energy of $\mathrm{V}_{\mathrm{o}}^{\cdot}[8]$. 
the largest ionic sizes, suppresses grain growth the most $[8]$.

Grain boundary energy was also found to correlate with the tendency for grain growth: tetragonal zirconia tends to have lower grain boundary energy (by 60\%) than cubic zirconia and hence lower grain boundary mobility [3-7]. The lower grain boundary energy could be a direct result of more solute segregation in tetragonal zirconia, which is consistent with the report that the amount of segregation in the cubic phase is less [10]. Moreover, according to the Borisov correlation [11], a higher grain boundary energy may be correlated to a higher grain boundary diffusivity in the cubic phase which will also enhance grain boundary mobility [7]. At any rate, the much higher energy of the cubic-cubic boundary and the cubic-tetragonal boundary gives rise to a large dihedral angle around $135^{\circ}$ (see Fig. 3). Thus, even at equilibrium, the cubic grain should be eight sided, and as shown in Fig. 3, is larger than the tetragonal grain in a two-phase tetragonal-cubic zirconia. This has been commonly observed.

A layer of siliceous glassy phase of several nanometer thickness often exists along the grain boundary of zirconia [12]. It is remarkable, however, that such a phase rarely seems to influence the properties of zirconia ceramics except in special cases (e.g. grain boundary electrical conductivity). In the present connection, we note that, despite the presence of such a liquid phase, characteristic dihedral phase angles between tetragonal and cubic grains have been observed [3]. Thus, grains are apparently communicating with each other across the thin liquid gap. In addition, even in the

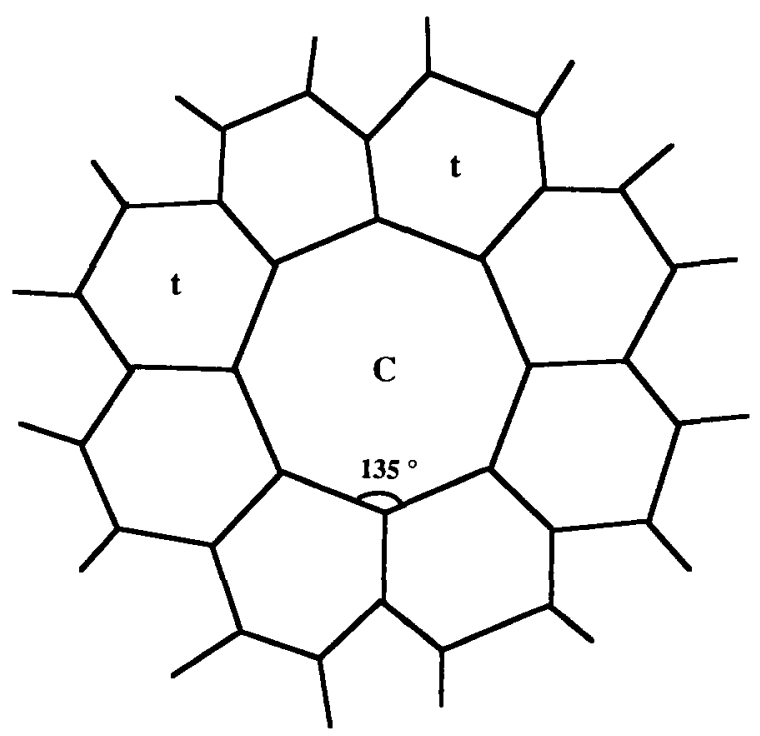

Fig. 3. Schematic diagram depicting a large, eight-sided cubic zirconia grain with a dihedral angle of $135^{\circ}$ surrounded by tetragonal zirconia grains. presence of a relatively large amount of liquid, around $5-10 \mathrm{v} / \mathrm{o}$, tetragonal grains still grow at a much slower rate than cubic grains $[13,14]$. Thus, the solute drag mechanism apparently persists at the solid-liquid boundary.

Although the grain boundary of tetragonal zirconia does not appear to have much anisotropy, and abnormal grain growth which is either due to a mobility advantage or an energy advantage rarely occurs, nonequilibrium conditions may arise to cause certain interfaces to have different energies and to induce anomalous migration. For example, diffusion-induced grain boundary migration (DIGM) has been reported in cubic zirconia which is related to the misfit elastic energy at the interface when excess solutes are deposited $[15,16]$. Enhanced grain boundary motion at selected boundaries, driven by the solute supersaturation but biased by the interfacial energy anisotropy, then proceeds as a result. Conversely, particle pinning has been found to be effective in retarding grain growth. For cubic zirconia, fine grained (grain size less than $1 \mu \mathrm{m}$ ) ceramics can be obtained by alumina addition [17]. For tetragonal zirconia, although the relatively low boundary mobility makes particle pinning unnecessary, some grain size refinement is still observed when a second phase is added (see Fig. 4).

Lastly, dynamic grain growth experiments in diffusional creep or superplastic deformation have confirmed the importance of grain boundary mobility. As shown in Fig. 5, the material with the lowest grain boundary mobility (Fig. 5(a)) has the lowest flow stress and the least strain hardening (Fig. 5(b)), and vice versa. Concurrent grain size measurement found the grain size to be stable during deformation for the low mobility material, and found rapid, strain-dependent

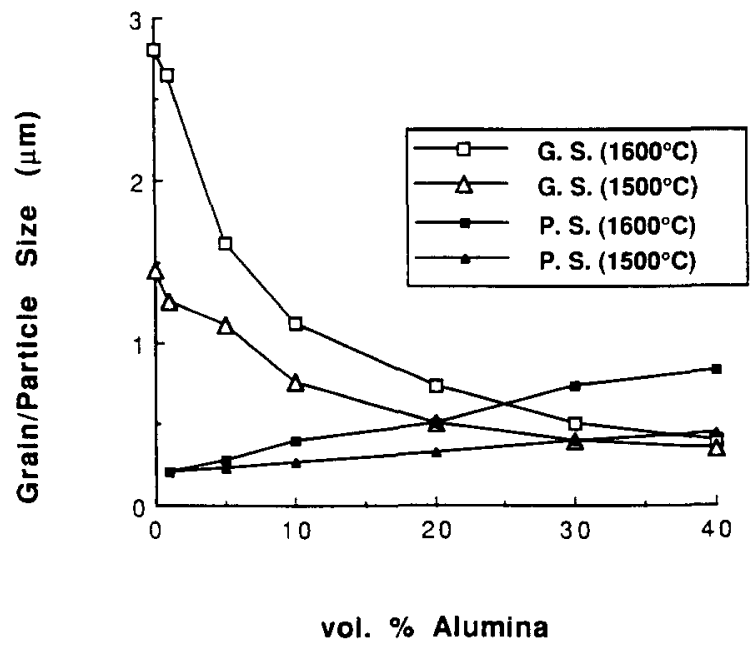

Fig. 4. Zirconia grain size (G.S.) and alumina particle size (P.S.) as a function of alumina content in a tetragonal zirconia stabilized by $12 \% \mathrm{CeO}_{2}$. Sintering temperatures are indicated in parentheses. 

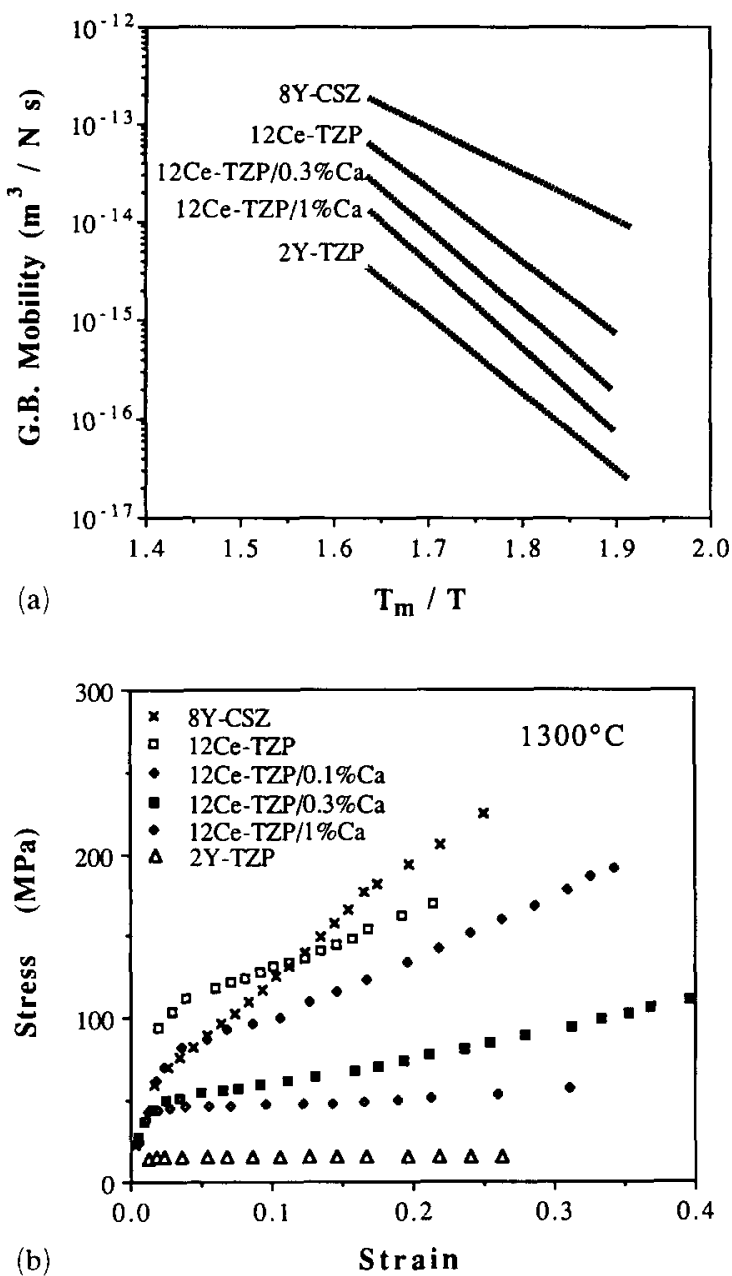

Fig. 5. (a) Grain boundary mobility of several tetragonal and cubic zirconia. (b) Stress-strain curves of the same set of materials [3].

grain growth in all the other materials. The fastest grain growth is observed in cubic zirconia under both static (annealing) and dynamic (deformation) conditions.

\section{Alumina}

Alumina is a stoichiometric ionic solid with very little solubility for aliovalent cations. Its crystal structure is of hexagonal type and is thus highly anisotropic. The grain boundary energy of alumina has been found to be even larger than that of cubic zirconia according to the grain/grain dihedral angle measurement [3]. Such characteristics of strong anisotropy and large grain boundary energy make alumina susceptible to rapid, and often exaggerated, grain growth. In addition, its behavior is sensitive to impurities since even a minor amount of additives will exceed its solubility limit.

It is well known that $\mathrm{MgO}$ has a beneficial effect on sintering of alumina; even a small addition of $\mathrm{MgO}$ is sufficient to suppress grain growth [18]. In ultrahigh purity alumina, $250 \mathrm{ppm} \mathrm{MgO}$ decreases grain boundary mobility by a factor of 50 at $1600{ }^{\circ} \mathrm{C}$ [19]. The same effect persists but is less dramatic in less pure alumina. The cause of this suppression has been attributed to solute drag [18]. However, direct confirmation of $\mathrm{Mg}$ segregation to the grain boundary has not been conclusive. Nevertheless, $\mathrm{MgO}$ does seem to reduce the boundary-to-boundary variation of segregation of other impurities (most notably $\mathrm{Ca}$ ) [20, 21], and variation of the grain boundary/surface dihedral angle [22], which will have a beneficial effect on preventing anomalous grain growth. However, $\mathrm{MgO}$ appears to increase boundary and lattice diffusivity of cations slightly [23], which could actually increase the mobility.

The impurity effect of the boundary is especially pronounced when alumina is accompanied by a liquid grain boundary phase. Liquid promotes grain faceting by facilitating shape equilibrium through enhanced kinetics. In addition, because the interface advances by depositing cations from the liquid, surface roughening is often discouraged owing to the large configurational entropy difference for cations in liquid and in solid [24]. Large platelets of alumina are therefore found in liquid-phase-sintered samples [25]. Impurities can further alter the energetics or kinetics across the liquid phase. In the first category, it is noted that charge compensating dopants (e.g. $\mathrm{TiO}_{2}+\mathrm{CaO}, \mathrm{SiO}_{2}+\mathrm{SrO}$, $\mathrm{SiO}_{2}+\mathrm{Na}_{2} \mathrm{O}[26]$, and $\left.\mathrm{TiO}_{2}+\mathrm{CuO}[27]\right)$ have been found to promote growth of elongated, plate-like abnormal grains, possibly by neutralizing the interface when they cosegregate. In the second category, it is noted that trivalent cations (e.g. $\mathrm{La}^{3+}, \mathrm{Y}^{3+}$ and $\left.\mathrm{Ce}^{3+}\right)$ increase grain boundary mobility and can even cause grain-particle (at $10 \mathrm{vol} . \%$ ) separation in a two-phase ceramic [28], see Fig. 6 . This dramatic effect is manifested, however, only when some $\mathrm{SiO}_{2}$ is present. Presumably, the liquid viscosity is lowered by these trivalent cations. The presence of alkali impurities seem to have the same effect [29]. Moreover, when a sufficient quantity of a scavenger that has large solubility for non-alkali cations is introduced, the enhancement effect is lost [28]. Thus, by introducing impurity and scavenger, the mobility of alumina can also be controlled to a good extent.

Returning to solute segregation, experimental evidence currently favors misfit strain energy as the main driving force for segregation of most divalent and trivalent cations [30]. With the dissolution of the dopants, charge compensation appears to occur in a self-compensating mode and no extended space charge layer has been observed. An exception may lie in tetravalent titanium substitution, however, which seems to achieve charge compensation by increased concentrations of negatively charged aluminum vacancies or oxygen 


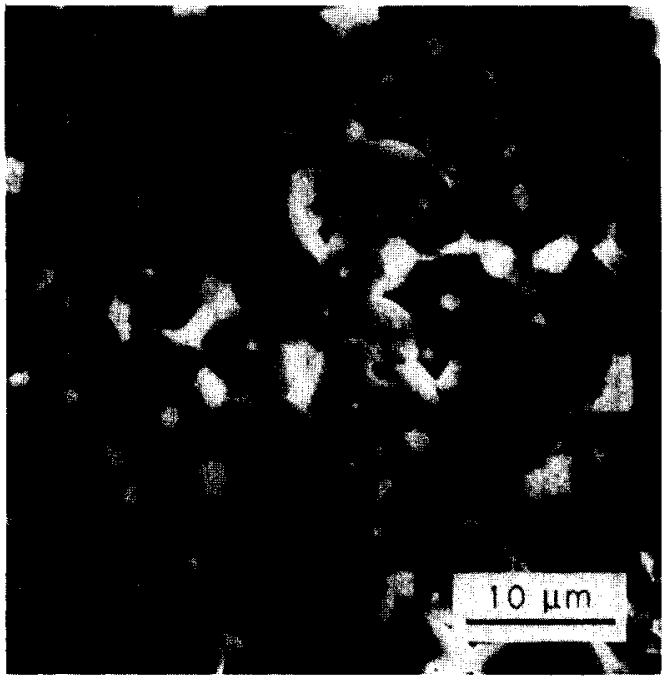

Fig. 6. Scanning electron micrograph of an alumina containing $10 \%$ second phase $\left(\mathrm{Zr}_{01.9} \mathrm{Ce}_{0.1} \mathrm{O}_{1.95}\right)$. Many particles have been separated from the grain boundary [28].

interstitials giving rise to a negative charge at the boundary. By the space charge mechanism, additional titanium segregation is then possible, which is in accord with the experimental observation. However, if the misfit mechanism is operative, a distinct dopant size effect on grain boundary mobility should be manifested. To our knowledge, this has not been demonstrated.

To maintain a very fine grain size in alumina, particle pinning is found most effective. Partially stabilized tetragonal zirconia, which has a remarkable resistance to grain coarsening as mentioned before, is an excellent additive for this purpose. The relationship between grain $\left(\mathrm{Al}_{2} \mathrm{O}_{3}\right)$ size and particle $\left(\mathrm{ZrO}_{2}\right)$ size has been studied by several investigators $[3,29,31,32]$. As shown in Fig. 7 , the results follow a linear relationship $d \sim 0.75 r / v^{1 / 3}$ where $v$ is the volume fraction of zirconia. This is equivalent to having a pinning particle every 2.5 grains, which is a much lower density than one particle at every four-grain junction (equivalent to six pinning particles every grain) as commonly envisioned.

As in the case of zirconia ceramics, the correspondence of static grain growth and dynamic grain growth, and the accompanying effect of grain boundary mobility on the stress-strain curve in diffusional creep-superplastic deformation, have been demonstrated in alumina $[3,33]$. To achieve a fine grain size which remains stable after large strain deformation, $\mathrm{MgO}$ doping and particle pinning were found necessary [34]. Otherwise, rapid dynamic grain growth occurs during deformation which causes severe strain hardening and large flow stress, as shown in Fig. 8. This results in premature specimen fracture.

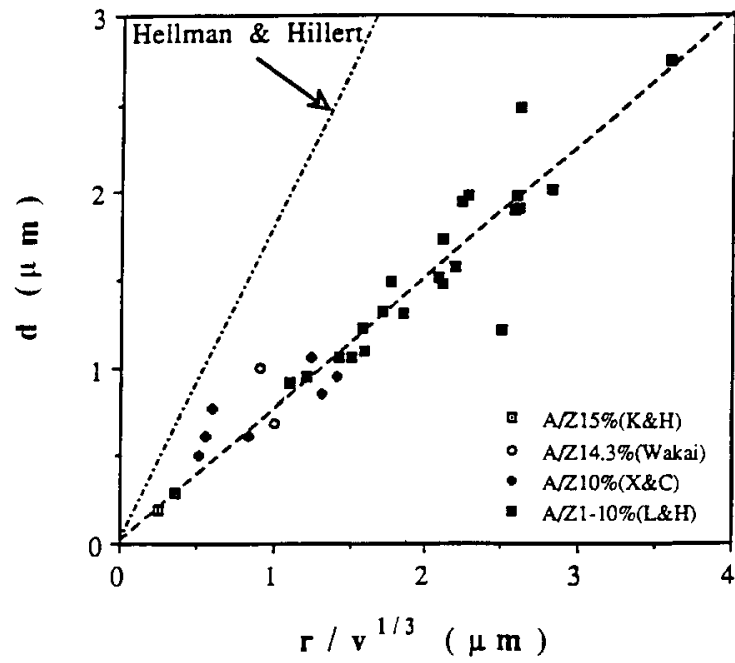

Fig. 7. Alumina grain size $d$ as a function of zirconia inclusion radius $r$ and volume fraction of inclusion $v$. References are $\mathrm{K} \&$ $\mathrm{H}$ [29], Wakai [32], X \& C [3], and L \& H [31].

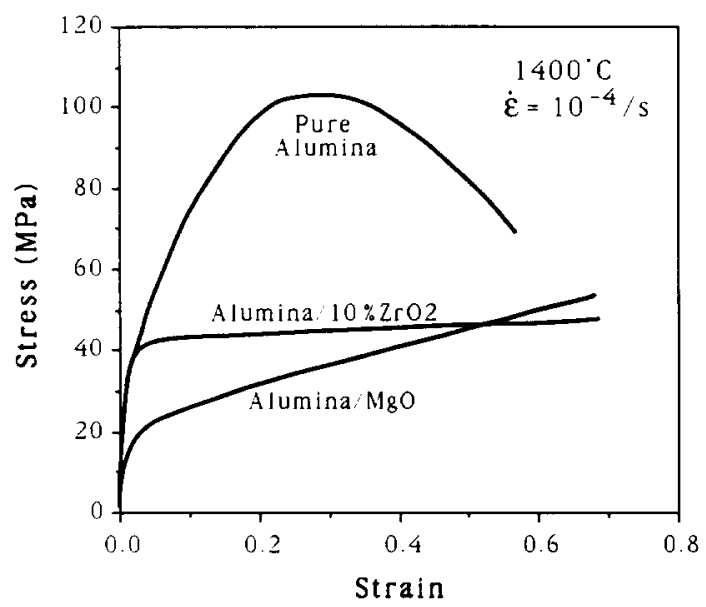

Fig. 8. Flow stress at a constant strain rate of $10^{-4} s^{-1}$ vs. strain curves for pure, $\mathrm{MgO}$-doped, and $\mathrm{ZrO}_{2}$-added alumina [34].

\section{Silicon nitride}

Silicon nitride and its solid solutions are covalent ceramics. To maintain charge balance, the $\mathrm{Si} / \mathrm{Al}$ and $\mathrm{N} / \mathrm{O}$ replacement reactions in $\beta^{\prime}$-SiAlON are always stoichiometrically related to each other. This requirement is relaxed somewhat in $\alpha^{\prime}-\mathrm{SiAlON}$ in which additional cations can be stuffed at the interstitial sites and participate in the charge compensation. These characteristics dictate that sintering of silicon nitride ceramics will not be assisted by diffusion of point defects (which do not exist) and must be conducted in the presence of liquid sintering aids. Since a large amount of liquid $(5-15 \mathrm{v} / \mathrm{o})$ typically is present, grain growth in silicon nitride should be regarded as Ostwald ripening and not 
a solid state grain boundary process [35]. The viscosity of the liquid, which is an oxynitride glass containing sintering aids, is of paramount importance in determining the rate of grain coarsening [36].

The most striking feature of silicon nitride ceramics is the shape anisotropy that is often observed for $\beta$ $\mathrm{Si}_{3} \mathrm{~N}_{4}$ and $\beta^{\prime}$-SiAlON. $\left(\alpha^{\prime} \mathrm{Si}_{3} \mathrm{~N}_{4}\right.$ and $\alpha$-SiAlON are always equiaxed except in the case of whiskers.) At smaller grain sizes, equiaxed $\beta$-Si $\mathrm{N}_{4}$ and $\beta^{\prime}$-SiAlON are more common, as exemplified by the superplastic $\beta^{\prime}$-SiAION [37]. Therefore, the shape anisotropy is mainly manifested in large grains, and it is the growth chracteristics that eventually enable long $\beta-\mathrm{Si}_{3} \mathrm{~N}_{4}$ and $\beta^{\prime}$-SiAlON rods to form in the microstructure.

The origin of this anisotropy has been recently explored using several empirical models [38]. First, by counting the broken bonds per unit area, it can be shown that the ratio of the specific surface energies of $\{1 \overline{100}\}$ and $(0001)$ faces is $1: 1.32$ in $\beta-\mathrm{Si}_{3} \mathrm{~N}_{4}$. This is a modest ratio and does not demand a high aspect ratio for the equilibrium grain shape. To estimate growth velocities, the periodic bond chain (PBC) theory may be invoked [39]. By estimating the energy release on attachment and growth of different faces, growth rates proportional to such attachment energies may be assigned. For example, it has been shown that $\{1100\}$ faces have the lowest attachment energy. According to the PBC theory, they should grow the slowest and constitute the surviving facets. Likewise, the $(0001)$ face has the highest attachment energy and should grow the fastest. These predictions are in agreement with the experimental observations [40]. Actually, the PBC theory also finds that $\{1 \overline{101}\}$ faces are slow growing and should become facets. These facets have been observed in $\beta-\mathrm{Si}_{3} \mathrm{~N}_{4}$ single crystals grown from a silicon melt or formed by a chemical vapor deposition process [41, 42]. By comparing the attachment energy of $\{1 \overline{1} 01\}$ faces and $\{1 \overline{1} 00\}$ faces, it can be shown further that the $\{1 \overline{1} 01\}$ faces should grow at a velocity four times that of $\{1 \overline{1} 00\}$. The growth form is thus a hexagonal prism with an aspect ratio around 4 , as shown in Fig. 9. In reality, the [0001] end surface of $\beta-\mathrm{Si}_{3} \mathrm{~N}_{4}$ or $\beta^{\prime}$-SiAlON tends to rounded [36], which suggests that a roughening transition has taken place for the $\{1 \overline{101}\}$ faces, as shown in Fig. 9. This has the consequence of allowing for faster growth rate by providing kink sites on the rough surface. Growth of $\beta-\mathrm{Si}_{3} \mathrm{~N}_{4}$ and $\beta^{\prime}$ SiAlON is thus highly anisotropic. Eventually, grain impingement takes place and the maximum aspect ratio before grain impingement is dependent on the growth time. Since the transverse growth of the atomically flat, prismatic $\{1 \overline{100}\}$ faces is very sluggish, the aspect ratio is also inversely dependent on the initial grain dimension which appears to be controlled by the particle size of the starting powders [43].
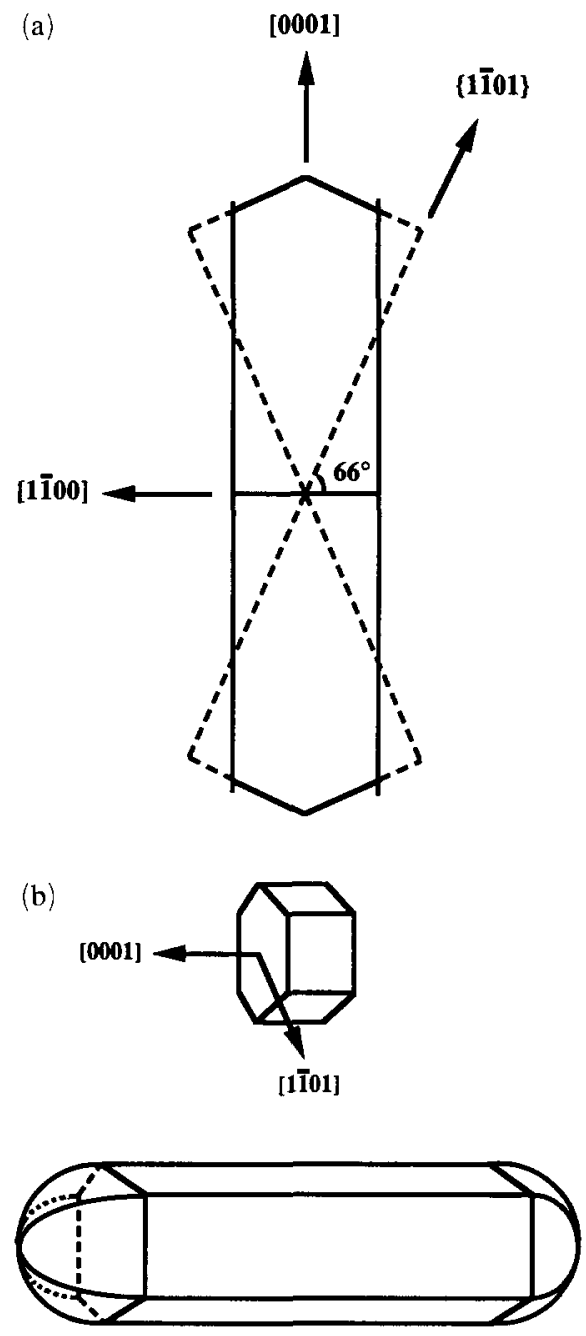

Fig. 9. (a) Predicted (PCB theory) grain shape (depicted by heavy lines) of $\beta-\mathrm{Si}_{3} \mathrm{~N}_{4}$ prism bounded by $(1100)$ and $\{1 \overline{1} 01\}$ faces. (b) $\beta$ - $\mathrm{Si}_{3} \mathrm{~N}_{4}$ prism with rough interfaces at the end.

Obviously, grain impingement is important and will ultimately limit the maximum aspect ratio achieved. If the grains are interlocked, despite the presence of a liquid, further grain growth onto the neighboring grains will strain the network by adding new matter to certain network segments by dissolution of others. The strain energy induced will probably reduce the driving force for grain growth. However, if the extent of grain interlocking is reduced, enhanced grain growth may be anticipated. Geometrically, this can be most efficiently accomplished by grain alignment in one direction, although even grain alignment in two dimensions is capable of reducing grain interlocking significantly. In agreement with this prediction, grain alignment in one and two dimensions, as in tensile tests [44] and biaxial stretching tests [38], respectively, has been found to enhance grain growth dramatically in the [0001] direction. This can be seen in Fig. 10. Some transverse inter- 


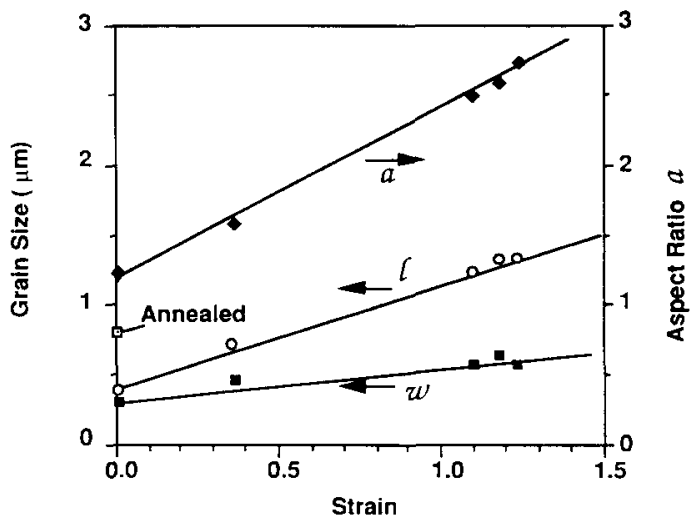

Fig. 10. Grain length $/$ width $w$ and aspect ratio $a$ vs. strain. The grain size obtained by annealing without deformation is also shown to the left $\mid 44]$.

growth also appears to have occurred among the unidirectionally aligned neighboring grains because of the proximity of their crystallographic orientations [44]. It is noted that the continuously evolving texture in these cases has a very pronounced effect on the deformation behavior of the material, as shown in Fig. 11 for a tensile stress-strain curve. This can be understood in terms of the microstructural features which resemble a short-fiber reinforced composite. Not surprisingly, large tensile ductility and biaxial formability have been achieved in such grain-aligned silicon nitride ceramics.

\section{Conclusions}

Grain boundary and interface boundary behavior varies vastly in zirconia, alumina, and silicon nitride. In zirconia, grain boundary mobility is usually isotropic, can be controlled by solute drag and is phase dependent. The presence of a liquid phase does not fundamentally alter the growth behavior. In alumina, grain boundary mobility is extremely sensitive to the presence of a liquid phase and the impurities within the latter. Anisotropic morphology is common but can be suppressed by the addition of $\mathrm{MgO}$ or impurity scavengers. In silicon nitride, Ostwald ripening is operational and the mobility of the solid-liquid interface is again phase dependent. The mobility of $\beta-\mathrm{Si}_{3} \mathrm{~N}_{4}$ is highly anisotropic leading to early grain impingement and strain effects that suppress subsequent growth. Finally, an additional enhancement of boundary or interface mobility is experienced when large strain, plastic deformation is imposed. This enhancement effect appears most pronounced in high mobility materials or those with highly interlocked microstructures.

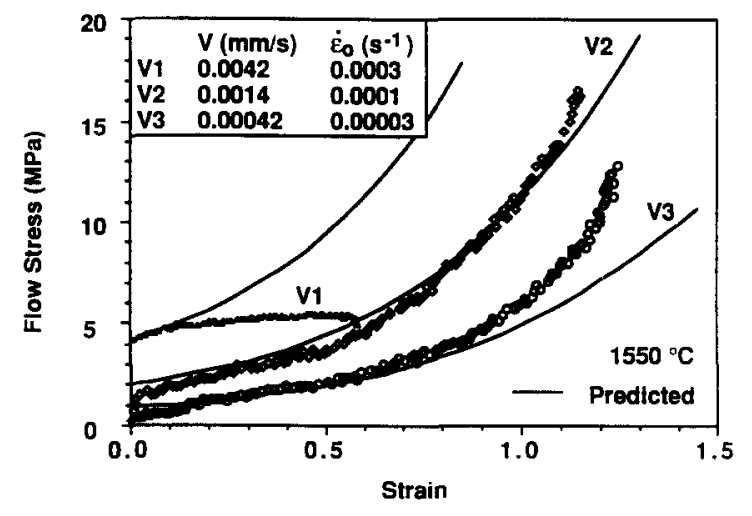

Fig. 11. Typical tensile stress-strain curves showing pronounced strain hardening at low strain rates due to grain alignment and enhanced grain growth. Also shown as solid curves are model predictions $[44]$.

\section{Acknowledgment}

I am indebted to my former and current students and coworkers whose research has contributed much to my understanding of grain growth. This review was prepared with the support of the US Department of Energy under Grant No. DE-FG02-87ER45302.

\section{References}

I R. J. Brook, Controlled grain growth. In F. F. Wang (ed.), Treatise on Materials Science and Technology, Vol. 9, Academic Press, New York, 1976, pp. 331-364.

2 R. S. Averback, H. J. Höfler and R. Tao, Mater. Sci. Eng. A, in press.

3 I-W. Chen and L. A. Xue, Development of superplastic ceramics, J. Am. Ceram. Soc., 73(9)(1990) 2585-2609.

4 D. M. Kroeger and A. Goyal, Critical currents and microstructures in oxide superconductors, J. Met., $44(10)(1992)$ $42-47$.

5 E. Tani, S. Umebayashi, K. Kishi, K. Kobayashi and $M$. Nishijima. Gas-pressure sintering of $\mathrm{Si}_{3} \mathrm{~N}_{4}$ with concurrent addition of $\mathrm{Al}_{2} \mathrm{O}_{3}$ and $5 \mathrm{wt} \%$ rare earth oxide high fracture toughness $\mathrm{Si}_{3} \mathrm{~N}_{4}$ with fiber-like structure. Am. Ceram. Soc. Bull., 65(9)(1986) 1311-1315.

6 T. K. Gupta. J. H. Bechtold, R. C. Kuzucki, L. H. Cadoff and B. R. Rossing, Stabilization of tetragonal phase in polycrystalline zirconia, J. Mater. Sci., 12(1977) 2421-2426.

7 I. G. Lee and I-W. Chen, Sintering and grain growth in tetragonal and cubic zirconia. In S. Somiya, M. Shimada, M. Yoshimura and R. Watanabe (eds.), Sintering 87, Proc. 4th Int. Symp. on Science and Technology of Sintering, Tokyo, November 4-7, 1987, Vol. 1. Elsevier Applied Science, London, 1988, pp. 340-345.

8 S.-L. Hwang and I-W. Chen, Grain size control of tetragonal zirconia polycrystals using the space charge concept, J. Am. Ceram. Soc, $73(11)(1990) 3269-3277$.

9 I-W. Chen, Solute drag on grain boundary in ionic solidsthe space charge effect. In R. Ishizaki and K. Nihara (eds.), Design and Control of Grain Boundary in Ceramics. Elsevier. New York, 1992, pp. 254-267. 
10 G. S. A. M. Theunissen, A. J. A. Winnubst and A. J. Burggraaf, Segregation aspects in the $\mathrm{ZrO}_{2}-\mathrm{Y}_{2} \mathrm{O}_{3}$ ceramic system, J. Mater. Sci. Lett., 8(1989) 55-57.

11 V. T. Borisov, V. M. Golikov and G. V. Schenbedinskiy, Phys. Met. Metallograph, 17(1964) 80.

12 M. Rühle, N. Claussen and A. H. Heuer, Microstructural studies of $\mathrm{Y}_{2} \mathrm{O}_{3}$-containing tetragonal $\mathrm{ZrO}_{2}$ polycrystals ( $\mathrm{Y}$ TZP). In N. Claussen, M. Rühle and A. H. Heuer (eds.), $A d v-$ ances in Ceramics, Vol. 12, Science and Technology of Zirconia II, American Ceramic Society, Westerville, $\mathrm{OH}$, 1984, pp. 352-370.

13 Y-J. Lin, P. Angelini and M. MeCartney, Microstructural and chemical influences of silicate grain-boundary phases in yttria-stabilized zirconia, J. Am. Ceram. Soc., $73(9)(1990)$ 2728-2735

14 M. MeCartney, Grain boundary phases in yttria-stabilized zirconia, Proc. Science and Technology of Zirconia V, Australian Ceramic Society, Melbourne, 1992.

$15 \mathrm{~J}-\mathrm{W}$. Jeong, D. N. Yoon and D. Y. Kim, Chemically induced instability at interfaces of cubic $\mathrm{ZrO}_{2}-\mathrm{Y}_{2} \mathrm{O}_{3}$ grains in a liquid matrix, Acta Metall. Mater., $39(6)(1991)$ 1275-1279.

$16 \mathrm{~J}$-W. Jeong, D. N. Yoon, J. Y. Lee and D. Y. Kim, Grain boundary migration in cubic zirconia-yttria induced by addition of magnesium at varying concentrations, J. Am. Ceram. Soc., $75(10)(1992) 2659-2664$.

17 J. D. French, M. P. Harmer, H. M. Chan and G. A. Miller, Coarsening-resistant dual-phase interpenetrating microstructures, J. Am. Ceram. Soc., $73(8)$ (1990) 2508-2510.

$18 \mathrm{~S}$. J. Bennison and M. P. Harmer, A history of the role of $\mathrm{MgO}$ in the sintering of $\alpha-\mathrm{Al}_{2} \mathrm{O}_{3}$. In C. A. Handwerker, J. E. Blendell and W. A. Kaysser (eds.), Sintering of Advanced Ceramics, Ceramic Transactions, Vol. 7, American Ceramic Society, Westerville, OH, 1990, pp. 13-49.

19 S. J. Bennison and M. P. Harmer, Grain growth kinetics for alumina in the absence of a liquid phase, J. Am. Ceram. Soc., $68(1)(1985) \mathrm{C} 22-\mathrm{C} 24$.

20 S. Baik and C. L. White, Anisotropic calcium segregation to the surface of alumina, J. Am. Ceram. Soc., $70(9)(1987)$ $682-688$

21 S. M. Mukhopadhyay, A. P. Jandine, J. M. Blakely and S. Baik, Segregation of magnesium and calcium to the $(10 \overline{10})$ prismatic surface of magnesium implanted sapphire, J. Am. Ceram. Soc., $71(5)(1988) 358-362$.

22 C. A. Handwerker, J. M. Dynes, R. M. Cannon and R. L. Coble, Dihedral angle $\varepsilon$ in $\mathrm{MgO}$ and $\mathrm{Al}_{2} \mathrm{O}_{3}, J$. Am. Ceram. Soc., $73(5)(1990) 1371-1377$.

23 R. M. Cannon and R. L. Coble, Review of diffusional creep of alumina. In R. L. Braat and R. E. Tressler (eds.), Deformation of Ceramic Materials, Plenum, New York, (1975), p. 61.

24 K. A. Jackson, Mechanism of growth. In Liquid Metals and Solidification, American Society for Metals, Cleveland, $\mathrm{OH}$, 1958, pp. 174-186.

25 W. A. Kaysser, M. Sprissler, C. A. Handwerker and J. E. Blendell, Effects of a liquid phase on the morphology of grain growth in alumina, J. Am. Ceram. Soc., 70 (5) (1987) 339-345.

$26 \mathrm{H}$. Song and R. L. Coble, Origin and growth kinetics of plate-like abnormal grains in liquid-phase sintered alumina, J. Am. Ceram. Soc., $73(7)(1990) 2077-2085$.
27 L. A. Xue and I-W. Chen, Low-temperature sintering of alumina with liquid-forming additives, J. Am. Ceram. Soc., $74(8)(1991) 2011-2013$.

28 L. A. Xue and $\mathrm{I}-\mathrm{W}$. Chen, Control of grain boundary pinning in $\mathrm{Al}_{2} \mathrm{O}_{3} / \mathrm{ZrO}_{2}$ composites with $\mathrm{Ce}^{3+} / \mathrm{Ce}^{4+}$ doping, J. Am. Ceram. Soc., 75 (4)(1992) 822-829.

29 B. W. Kibbel and A. H. Heuer, Exaggerated grain growth in $\mathrm{ZrO}_{2}$-toughened $\mathrm{Al}_{2} \mathrm{O}_{3}, J$. Am. Ceram. Soc., $69(3)(1986)$ 231-236.

30 C. W. Li and W. D. Kingery, Solute segregation of grain boundaries in polycrystalline $\mathrm{Al}_{2} \mathrm{O}_{3}$. In W. D. Kingery (ed.) Structure and Properties of $\mathrm{MgO}$ and $\mathrm{Al}_{2} \mathrm{O}_{3}$ Ceramics, Advances in Ceramics, Vol. 10, American Ceramic Society, Westerville, $\mathrm{OH}, 1984$, pp. 368-378.

31 F. F. Lange and M. Hirlinger, Hindrance of grain growth in $\mathrm{ZrO}_{2}$-toughened $\mathrm{Al}_{2} \mathrm{O}_{3}, J$. Am. Ceram. Soc., $67(3)(1984)$ 164-168.

32 F. Wakai, T. Iga and T. Nagano, Effect of dispersion of $\mathrm{ZrO}_{2}$ particles on creep of fine-grained $\mathrm{Al}_{2} \mathrm{O}_{3}, J$. Am. Ceram. Soc. $96(12)(1988) 1206-1209$

33 L. A. Xue and I-W. Chen, Deformation and grain growth at low temperature-sintered high-purity alumina, $J$. Am. Ceram. Soc., $73(11)(1990) 3518-3521$.

34 L. A. Xue and I-W. Chen, Superplastic alumina ceramics with grain growth inhibitors, J. Am. Ceram. Soc., 74 (4) (1991) 842-845.

35 D. D. Lee, S. J. L. Kang and D. N. Yoon, Mechanism of grain growth and $\alpha-\beta^{\prime}$ transformation during liquid-phase sintering of $\beta^{\prime}$-SiAlON, $J$. Am. Ceram. Soc., 71 (9) (1988) 903-906.

36 C. M. Hwang, T. Y. Tien and I-W. Chen, Anisotropic grain growth in final stage sintering of silicon nitride ceramics In S. Somiya, M. Shimada, M. Yoshimura and R. Watanabe (eds.), Sintering 87, Proc. 4th Int. Symp. on Science and Technology Sintering, Tokyo, November 4-7, 1987, Vol. 2, Elsevier Applied Science, London, 1988, pp. 1034-1039.

37 I-W. Chen and S.-L. Hwang, Shear thickening creep in superplastic silicon nitride, J. Am. Ceram. Soc., 75 (5) (1992) 1073-1079.

38 S.-L. Hwang, Fabrication, microstructural characterization, and deformation of superplastic SiAlON ceramics, PhD Dissertation, Department of Materials Science and Engineering, University of Michigan, Ann Arbor, MI, 1992.

39 P. Hartman, Structure and Morphology. In P. Hartman (ed.) Crystal Growth: An Introduction, North-Holland, Amsterdam, 1973, pp. 367-402.

$40 \mathrm{P}$. Drew and M. H. Lewis, The microstructure of silicon nitride ceramics during hot-pressing transformation, $J$. Mater. Sci., 9(1974) 261-269.

41 R. Grun, The growth of $\beta-\mathrm{Si}_{3} \mathrm{~N}_{4}$ single crystals, J. Cryst Growth, 46(1979) 136-146.

$42 \mathrm{Y}$. Inomata and $\mathrm{T}$. Yamane, $\beta-\mathrm{Si}_{3} \mathrm{~N}_{4}$ single crystals grown from Si melts, J. Cryst. Growth, 21 (1974) 317-318.

43 K. R. Lai, Grain growth in $\beta$-SiAlON, PhD Dissertation, Department of Materials Science and Engineering, University of Michigan, Ann Arbor, MI, 1992.

$44 \mathrm{X}$. Wu and I-W. Chen, Exaggerated texture and grain growth of a superplastic SiAION, J. Am. Ceram. Soc., 75(10)(1992) 2733-2741. 